\title{
GUÍA PARA CREAR UN OBJETO DE APRENDIZAJE INCLUSIVO, BASADO EN MEDHIME
}

\section{GUIDE TO CREATE AN OBJECT OF LEARNING INCLUSIVE BASED ON MEDHIME}

\author{
María Eugenia Moreira ${ }^{1}$
}

Recibido para publicación: 14 de Agosto 2014 - Aceptado para publicación: 16 de octubre 2014

\section{RESUMEN}

A través de éste documento explicaremos algunas estrategias inclusivas utilizadas en el Centro Educativo manejando como apoyatura el Objeto Virtual de Aprendizaje "Atención Temprana", diseñado con MeDHiME y se construyó una estructura pedagógica sobre los ítems a tener en cuenta para aplicar la Inclusión Educativa desde la Atención Temprana (niños de 0 a 3 años) hacia las aulas en escuelas convencionales [1].

Palabras Claves: aulas inclusivas, ayudas para la comunicación, sistemas adaptativos, tecnología educativa, trabajo en equipo

\begin{abstract}
Through this document explain some inclusive strategies used in the Education Center apoyatura driving like the Virtual Learning Object "Early Warning", designed with MeDHiME and pedagogical structure was built on items to consider implementing Inclusive Education from Early Care (children 0-3 years) to the classes in regular schools.
\end{abstract}

Keyboards: Inclusive classrooms, communication aids, adaptive systems, educational technology, teamwork.

1(euge.trabajo@gmail.com) - Técnica Universitaria en Sistemas de Información. Universidad Nacional de San Juan - Argentina. Tel. 542644239123 


\section{INTRODUCTION}

Especificaremos el concepto de Inclusión (la figura 1 muestra el logo adoptado): se aborda desde el concepto de respetar el derecho de los niños y jóvenes a recibir una educación de calidad en entornos de igualdad y diversidad, una diversidad que contempla las diferentes características del alumnado, sus necesidades individuales, sus diferentes pautas de comportamiento o sus variados estilos de aprendizaje [2]. Tomando como punto de partida el Diseño centrado en el Usuario.

El Diseño centrado en el Usuario se relaciona con un heterogéneo conjunto de metodologías y técnicas que comparten un objetivo común: conocer y comprender las necesidades, comportamiento y características del usuario[3]. El gráfico de Inclusión está disponible en la siguiente dirección de página web: http://www.argentinareal.org/wpcontent/uploads/2012/12/exclusion.jpg

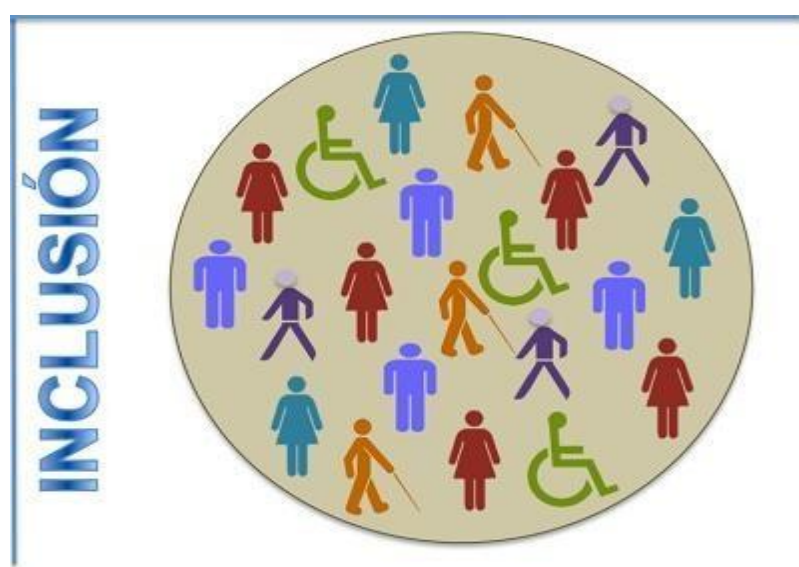

Fig. 1 - Logo de Inclusión

\section{SPECIFICATIONS FOR LEARNING OBJECTS AND ACTIVITIES}

Trataremos el concepto de "Atención Temprana" a través de un Objeto Virtual de Aprendizaje Inclusivo en donde se aplicó la esencia del concepto, destinado a Padres, Terapeutas y Profesionales idóneos de Atención Temprana.
Se insertaron videos subtitulados para que estén disponible a todas personas y que a la vez un lector de pantalla lo reconozca.

Se eligió la técnica grupal en donde a través de Grupos de Trabajo se consensuan las diferentes opiniones en cuanto al Aprender a Ser[4], que es una característica necesaria para poder observar y plasmar de forma escrita las fortalezas individuales de la personalidad relacionada con las expectativas personales en cuanto a la Inclusión de la Persona en el ambiente familiar y profesional. Luego en la etapa de evaluación posterior a observar un video en donde se muestran las estrategias de los adultos hacia los niños se comienza con una lluvia de ideas, acerca de los conceptos, experiencias y recursos referidos a la Atención Temprana en donde a través de un trabajo grupal se consensuan las opiniones del grupo.. Las escuelas inclusivas deben reconocer y responder a las diversas necesidades de los estudiantes, arreglos tanto en diferentes estilo s como al ritmo del aprendizaje y asegurando la calidad de la educación par a t o do s por medio de un currículo apropiado , dando lugar tanto a arreglos organizacionales, estrategias de enseñanza, uso de recursos y asociaciones con sus comunidades [5] .

Las mejores prácticas inclusivas:

- observar las características de los alumnos incluidos (o excluidos).

- visualizar de forma general los retos dentro del proceso educativo, como en el caso de la Atención Temprana que los niños de 0 a 3 años puedan desarrollar la motricidad fina y gruesa, autonomía e interacción social con los demás niños y desarrollo cognitivo.

- centrarse con más detalle en las intervenciones y prácticas áulicas inclusivas; es decir; en el caso de la Atención Temprana pautar entre Profesionales y Padres coordinadamente tanto en la Institución como en el ambiente familiar cuando y de que forma se debe intervenir para evitar que la autonomía del niño 
disminuya; definir los límites dados con cariño y aprecio a través de una voz suave y firme en cuanto a las tareas diarias que debe realizar el niño.

A partir de diferentes preferencias y necesidades (originadas por el propósito de la actividad de trabajo o aprendizaje y, por supuesto, de la naturaleza de los propios usuarios) se puede seleccionar el medio de representación más apropiado o conseguir la información a través de una amplia gama de medios de representación.(pictogramas, videos subtitulados, música que son los ítems a realizar como actividad en un Objeto Virtual de Aprendizaje Inclusivo, estén bien definidos y concretos, que no existan elementos que llamen la atención en cuanto a colores muy fuertes o demasiado impactantes).

Para construir Objetos Virtuales de Aprendizaje Inclusivos se aplicó los siguientes factores:

- Enseñanza Cooperativa: en las instituciones se relacionan los diferentes profesionales dentro y fuera del Centro Educativo dirigiendo el Proceso de Inclusión Educativa en las escuelas convencionales desde Nivel Inicial hasta el Nivel Secundario[6].

- Aprendizaje Cooperativo: trabaja la Docente Común con la Docente de Apoyo con la guía de las diferentes profesionales del Centro Educativo que pertenecen al Gabinete Pedagógico como psicólogas, psicopedagogas, asistente social, fonoaudiólogas; relacionadas a las estrategias y prácticas de aulas inclusivas[6].

- Resolución Cooperativa de problemas: en el Centro educativo se aborda reglas de comportamiento claras relacionadas a los niños y una serie de límites acordados con los Padres y Profesionales[6].

- Agrupamientos heterogéneos: se agrupan alumnos sin discapacidad y alumnos con discapacidad en el aula .Desde la Atención Temprana se les recomienda que los niños tengan actividades y relaciones personales con otros niños del mismo grupo etario e incluso de otro grupo etario mas grande para transmitirle de alguna forma el concepto de autoridad. Se debe poseer de objetivos programados y de rutas alternativas para el aprendizaje y la enseñanza personalizada y flexible[6].

- Enseñanza efectiva: se debe tener en cuenta la enseñanza- aprendizaje con una instrucción directa reflejada en las consignas que le apliqué en el Objeto Virtual de Aprendizaje , como en el caso de la Atención Temprana mostrar la esencia de éste concepto sin tener en cuenta los detalles superfluos. Pensar en una evaluación sistemática del progreso del alumno / a en diversas áreas (cognitiva, emocional, social, relacional, etc.) proponiendo medidas para superar las dificultades[6].

El equipo de desarrollo y diseño debe ser equilibrado:

- Se han de cubrir todos los aspectos: desarrollo, expresión, representación, factores humanos, usabilidad, técnicas pedagógicas aplicadas a la Diversidad.

- El trabajo en equipo ha de caracterizarse por la posibilidad de una comunicación e interacción rápida y efectiva.

El diseño debe caracterizarse por su simplicidad.

El uso del diseño ha de ser fácil de entender, independientemente de la experiencia, conocimiento, capacidades lingüísticas o nivel de concentración del usuario.

Hay que controlar la información explicitada, que se ha de reducir al mínimo necesario.

Se debe utilizar diversos recursos y estrategias educativas diferenciadas que atienda a las necesidades individuales de cada educando 


\section{CONCLUSION}

Teniendo en cuenta los factores mencionados anteriormente se visualizó a través de experiencias acumulada en cuanto a la Inclusión que se debe construir un Objeto Virtual de Aprendizaje Inclusivo y que los resultados arrojados fueron que todo el alumnado del grupo etario pudo aprender, esto implicó utilizar estrategias de aprendizajes de modo tal que contemple los diferentes modos de aprender que se manifestaron desde preferencias individuales hasta profundas necesidades individuales del alumno.

\section{REFERENCIAS}

[25]. Sirvente, F.A. "Lifting Académico-Tus clases fácil y rápido en Internet con MeDHIME 2.0" - Editorial de la Fundación de la Universidad Nacional de San Juan ISBN 978-950-605-709-1. Año 2012

[26]. http://pdi.cnotinfor.pt/?font=Arial\&color $=1$ \& size $=100 \& l a$ ng=1\&mode=list\&categ=2\&type=actionare

a

[27]. http://www.congresointernetdelmediterrane o.com/present aciones/2011/MK/torresburriel.pdf

[28]. http://www.elciudadano.cl/2014/01/09/101 567/aprendera-ser-la-dimensionolvidada-de-la-educacion/

[29]. [http://www.irisproject.eu/teachersweb/ES/ docs/TT_Estrat egias_y_Practicas_en_Las_Aulas_Inclusiv as_WD_ES.pdf

[30]. http://www.europeanagency.org/sites/default/files/inclusiveeducation-and- classroom-practices_iecpes.pdf 